\title{
Smart Parking with Fair Selection and Imposing Higher Privacy Constraints in Parking Owner and Driver Information
}

\section{B. Vivekanadam}

Senior Lecturer, Faculty of Computer Science and Multimedia, Lincoln University College,

Malaysia

E-mail: vivekanandam@lincoln.edu.my

\begin{abstract}
The use of private cars has enhanced the comfort of travel of individuals, but has proven to be a challenge for parking in congested downtown areas and metropolitans. This hike in the vehicle count has led to difficulty among the drivers to find a parking spot, exploiting resources and time. On the other hand, there are many idle private parking spots that remain inaccessible because of multiple reasons like unavailable owners, different open timings and so on. In order to prevent parking issues as well as to enable the use of private parking spots, smart parking applications that are easy to use by the drivers will prove to be highly effective. However, most parking lot owners and drivers face the threat of privacy which affects their willingness to participate while many others are located in a centralized location where the presence of malicious users is in plenty. In this proposed work, we have introduced a smart-parking system that is based on blockchain exhibiting qualities of privacy protection, reliability and fairness. To protect the privacy of users, vector-based encryption, bloom filters and group signatures are also insisted. This has helped us establish a more reliable smart parking system coupled with fair operation for smart contact. Experimental analysis of the real-world dataset indicates that the proposed work operates with high efficiency, establishing privacy protection, reliability and fairness.
\end{abstract}

Keywords: Privacy protection, smart parking, block chain, vehicular network, reliability 


\section{Introduction}

In today's world, the luxury of transport has become a common level of comfort due to the economic affordability of vehicles which has led to increase in buying of vehicles. The biggest drawback involved is the difficulty in finding an apt parking spot in congested metropolitan areas. According to a survey taken in 2020, there is need for more than a million parking spots in Beijing and over 50 million parking spots in China [1]. This has led to an unnecessary time wastage caused when drivers drive around looking for a space to park, often known as cruising. This process is time consuming and is also associated with wastage of resources such as fuel and energy. Similarly, another survey indicates that about $40 \%$ of the traffic congestion is caused due to the holdup caused when drivers are looking for a proper parking spot. Studies indicate that this will result in a delay of $8.2 \mathrm{~mm}$ which causes 696 tonnes of carbon dioxide emission, 50,000 extra gasoline burned and 950,000 added miles in Los Angeles for a period of 1 year. However, there are many private parking spots that remain idle and unused in many places [2]. These places include residential areas where the need for the parking spots is considerably less. Consider a parking owner who works for 8 hours a day, during which time he will not be available at home and will be taking his vehicles to reach his work place. During these 8 hours, his parking spot at home remains free. This indicates that there are many parking spots that are idle during various instances of time which when used effectively will prove to be beneficial for other users who are looking for parking spots in that area or nearby location. This method will also prove to be effective in giving the owners a financial benefit to meet some of their maintenance cost [3].

Though this proves to an efficient and simple solution, it leads to several privacy issues that need to be immediately addressed. If one can access the information about a parking spot and the driver's parking request, through a common cloud server, it will open ways in which malicious adversaries and untrustworthy servers' access information that are sensitive in nature. Hence this places a constraint on the driver as well as the parking owner, proving to be a difficult aspect to satisfy in establishing such a harmonious network. To address the issue of privacy in some smart parking, many smart parking schemes that adhere to privacy preserving have been introduced which hides the exact location and identity of both parking owners as well as driver [4]. These small-schemes that are established privately cannot be application or used in smart parking scenarios at a large scale. Moreover, some schemes 
involve the use of distributed Road-Side Units or centralized cloud server to save the details about drivers and parking spots which makes it easy to manage information. Despite these precautions, there is still need for better privacy improvements to enhance the privacy level at different entities [5-6].

Some challenges include large bandwidth that is essential in a centralized server design in order to work in a more efficient manner. However, when using such a system, it is possible for the entire system to come to a halt when the server fails [7]. These attacks and failures of the server might also indicate incorrect matches. Similarly, it is not possible to have centralized computer with large computational capacities or storage such as those available in nodes and cloud servers when distributed RSU model is involved making it unsuitable for parking operation. Moreover, they will also need inter-communication for wider system coverage. A typical VANET will not be able to support high communications as it might lead to congestion in the network. Hence both distributed RSU and centralized servers cannot meet the demands of smart parking schemes [8]. Apart from these limitations, many other schemes proposed over the years do allot parking spots in a fair manner. Some issues involved in a typical smart parking system that requires a 'fair' means of judging are:

- Parking owners might show information of fake parking spot in order to increase their income

- Drivers who are parking might refuse to pay their parking fees

Due to these aspects, it is not possible to provide a reliable smart parking service [9-10]. Hence, users who are opting for the smart parking service might not get their payment's worth of parking making the system unfair in many circumstances. In order to address these shortcomings, it is necessary to create a fair means of smart parking system that will take into consideration the hinderances and discrepancies of both the parking space owner as well as the parking driver. The proposed work uses a decentralized privacy-preserving parking scheme that ensures reliability and fairness [11]. 


\section{System Mode}

There are five elements in the proposed smart parking system as shown in the Fig.1.

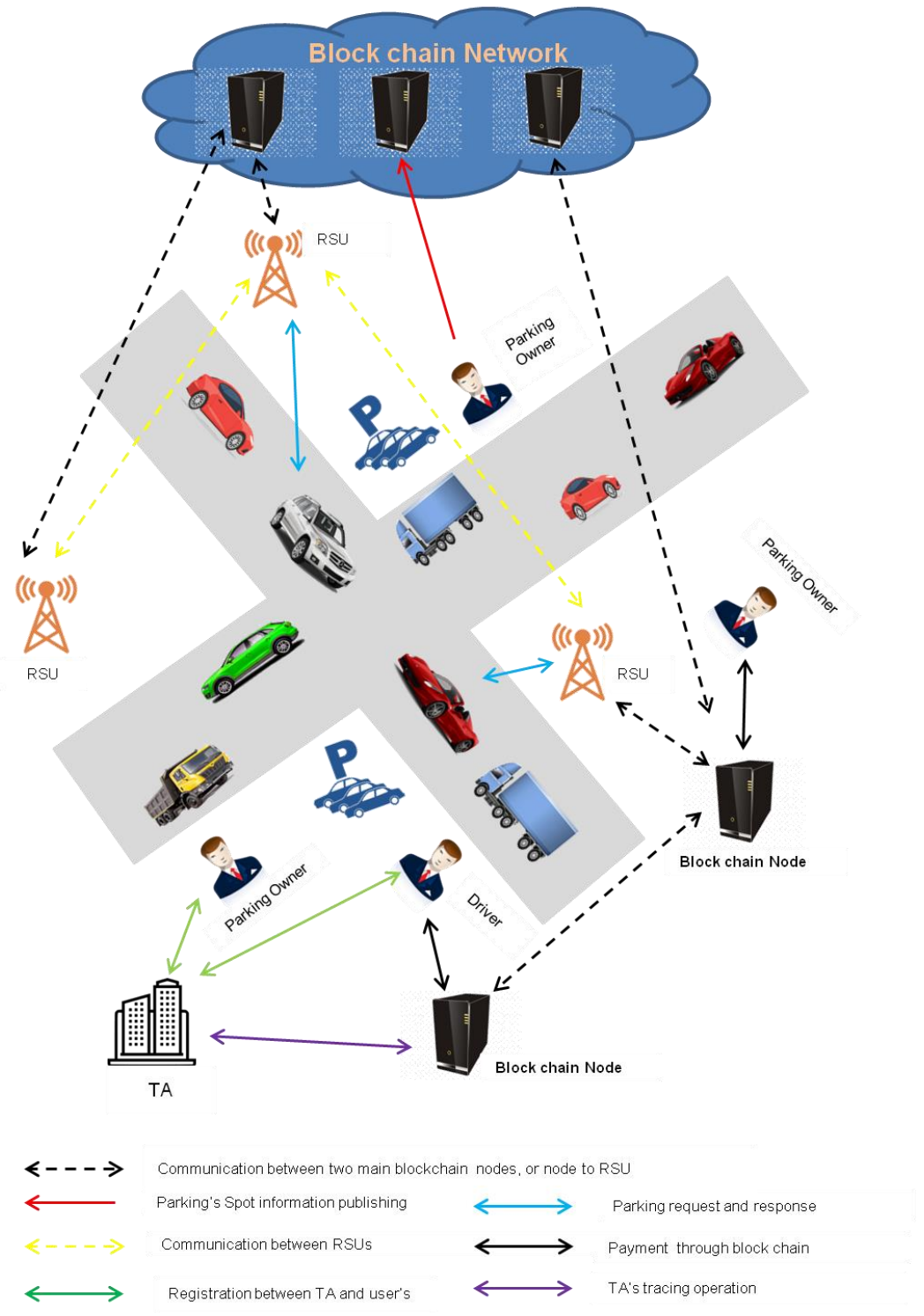

Fig.1. System Model of the Proposed Work

1. Blockchain: In this work, block chain technology is used to encrypt the parking requests and store them in a reliable manner [12]. Along with this, the parking request can also be managed in a more efficient manner using smart contract which gives a parking spot matching result as the return. Fair and anonymous parking payment is established using blockchain technology.

2. RSU: The RSU [13] proposed in our work exhibits a crucial task between the blockchain network and the driver, serving as the intermediary node. When the parking request from the 
driver is received, it is authenticated. If proved to be true, this is further encrypted using time vector to create a new parking request which is then sent to the blockchain. Moreover, it will also send the results of parking spot machine to the driver.

3. Driver: This work is carried out based on the assumption that every driver is driving Electric Vehicles and is thereby able to receive as well send transactions. If a driver decides to park nearby, a request will be sent to the nearest RSU. Once the result is received by the driver regarding the parking owner, the driver will initiate request of a parking spot [14]. On confirmation from the parking owner, the driver will receive the parking location.

4. Parking Owner: The owner of an idle parking spot can allow it for rent to generate income. To participate in this system, the owner should initially verify their identity by registering and further provide details of the parking spot. When the parking spot is used, the driver using it will pay parking fees.

5. TA: At the beginning phase, many keys and public parameters are generated for every driver and parking owner. On initialization, it will not be used unless a dispute arises. In such case, it is possible to find the actual identify of the parking owner or driver who misbehaves with the help of the TA. Hence the use of TA will not alter or change the way in which the system operates. Rather, it enables further security of the decentralized property.

\section{Design of the Fair and Smart Parking (FSP) Scheme}

In this work, a fair and smart parking system is proposed such that it provides a secure means of parking and is also reliable. There are four major phases involved in this system: initialization, parking spot identification, request and allotment.

1. Initialization: In this step, the shared keys are initially generated along with security parameters. Every parking lot owner is allowed keys sent by the TA using public-key cryptosystem using digital sign. In order to obtain the data, both the drivers and parking owners should submit their identity proof to the TA. This way the TA can also keep track of malicious and misbehaving users in case of disputes and mishaps

2. Publishing the Parking Spot: In this step, the information about parking spots that are available for use are defined by the parking owners. This information is fed into the system where it the location is identified based on segregation of the neighbouring areas based on grid pattern as shown in fig.2. To protect the privacy of location, the parking spot location is initially converted into grid coordinates that are correlated with a particular area within a particular city. Similarly, protection of time availability is also made using time vector is also 
accomplished. For the purpose of transactions, blockchain is used to main anonymity of the account.

3. Request of Parking: When the car parking is intended a request is sent by the driver to the blockchain as an encrypted text. A location range of the request is identified and grids are determined to find a suitable parking spot for the driver. On identification, parking time is next determined and encoded using a process similar to that of the owner.

4. Identification of Parking Spot: When a parking request of the driver is received, the RSU is generated and the signature of the driver is verified. Smart contract is obtained after successfully verifying the data. Based on the encrypted grid location and partitioned area, it is possible to determine the encrypted parking time and grid location range. There are two steps involved in this phase: location identification and time identification.

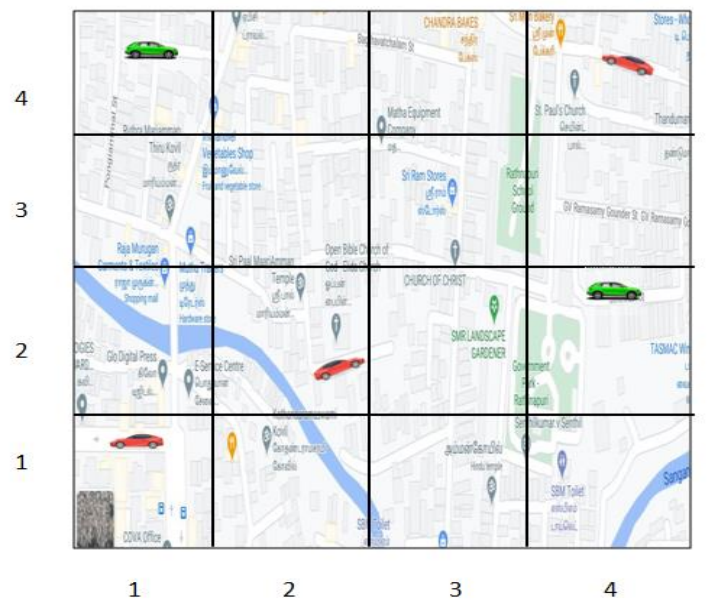

Fig.2. Grid Arrangement of Location

\section{Results and Discussion}

The four processes of the proposed FRP scheme is analysed and it has been found that in the initialization process, as the number of entities increases, the time taken for execution also increases subsequently as shown in Fig.3. Similarly, Fig.4 represents the average execution time cost to publish and verify as well as the time taken for encryption process. 


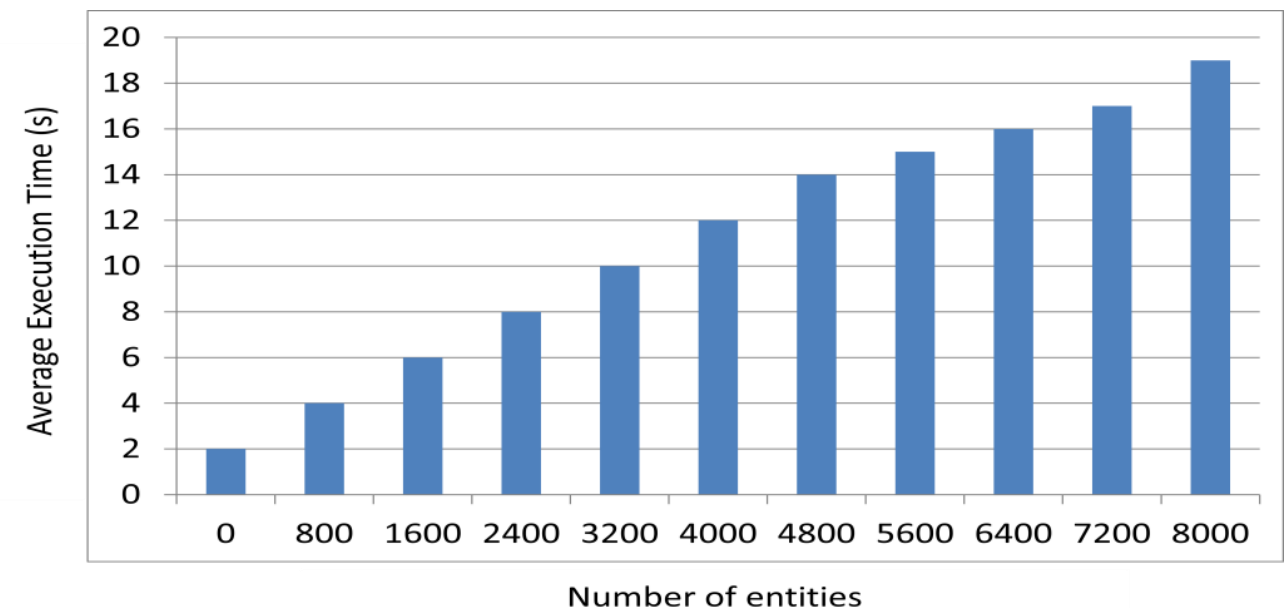

Fig.3. Initialization

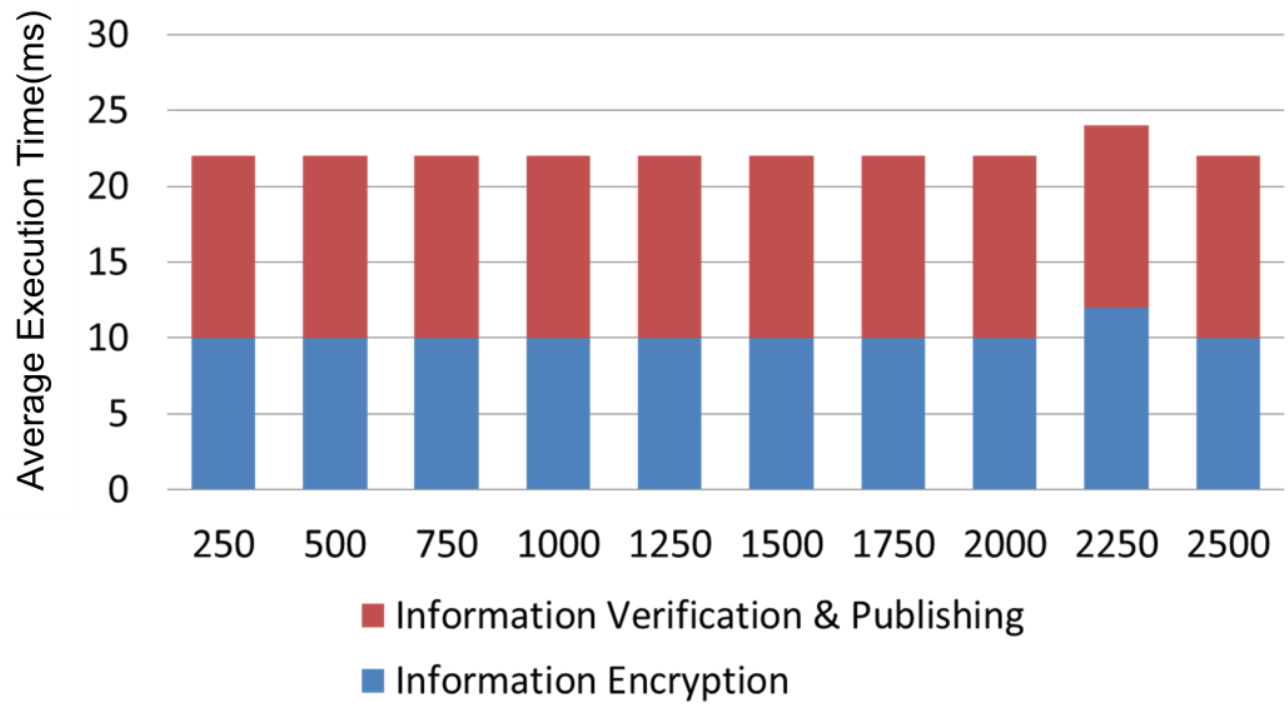

Number of Parking owner's

Fig.4. Publishing the Parking Spot

When a parking spot needs to be identified by the driver, a parking request is initially generated which consists of an encrypted time vector and location search token. This takes place independent of the drivers, as shown in Fig.5. In Fig.6, observation indicates that the average execution increases as the parking owners increases. 


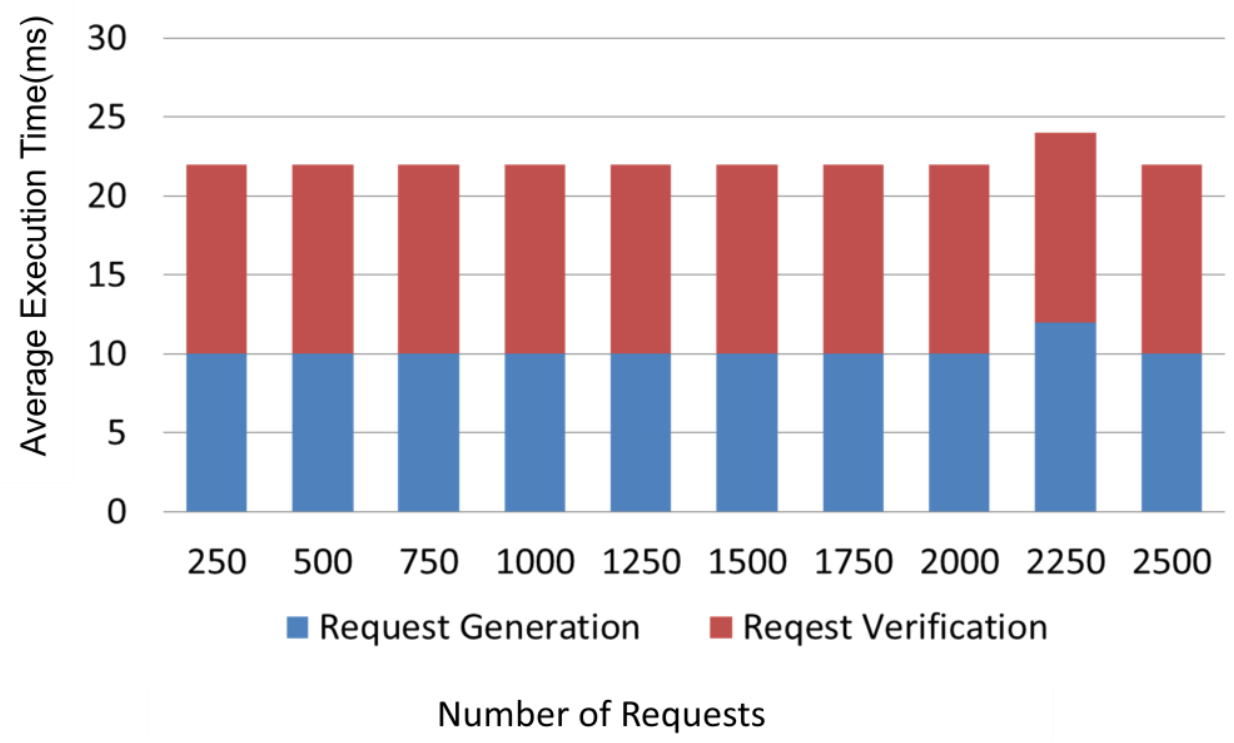

Fig.5. Request of Parking

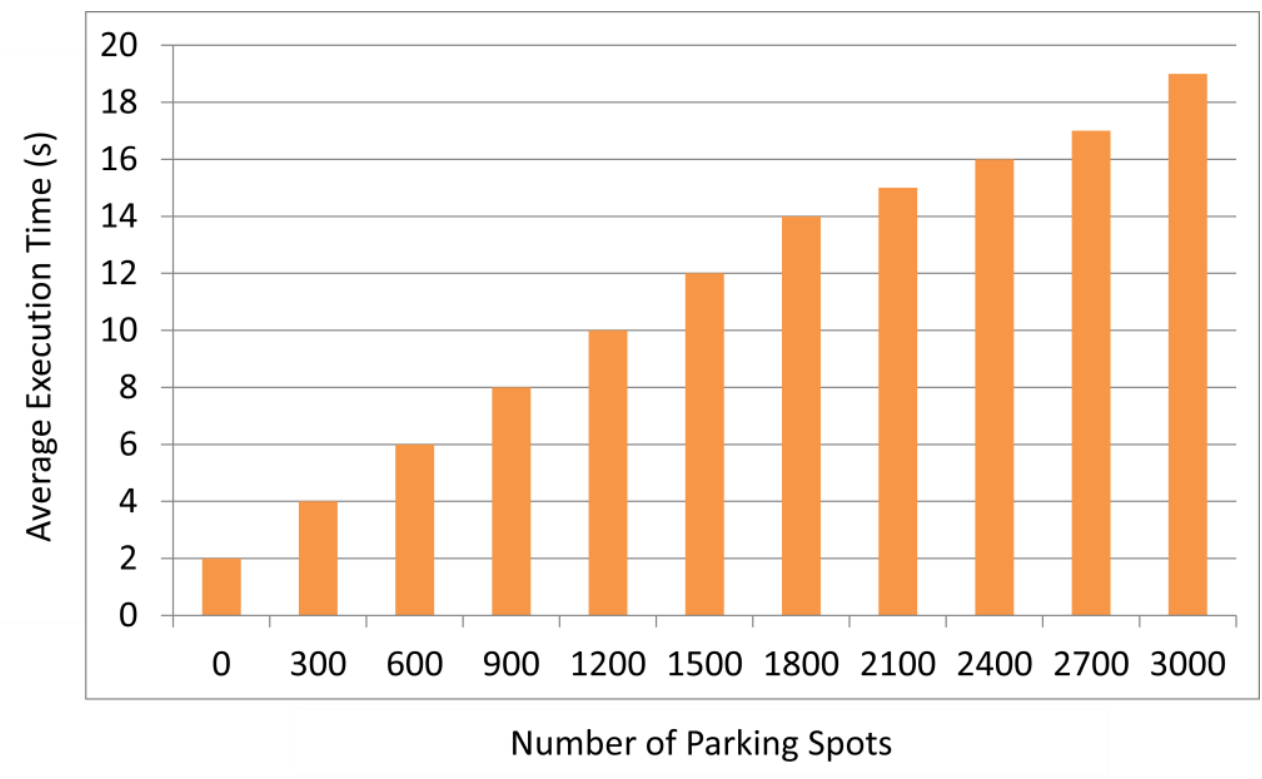

Fig.6. Identification of Parking Spot

\section{Conclusion}

In this proposed model, a novel system of smart parking that uses a reliable and fair means of parking is incorporated with emphasis on secure information exchange. A blockchain-based parking methodology is developed using vector-based encryption, bloom filter, secure pseudo-random function and BBS group signature in order to ensure security of both the parking owners as well as the drivers. TA enables traceability to the parking owners and the driver for access during mishaps. Fairness is achieved through smart contract and the 
proposed schemes ensures security of data privacy, time privacy, location privacy and identity privacy. Experimental observations of the proposed indicate traceability, authentication, reliability and integrity of the system. When implemented in real-time world dataset, a higher efficiency in communication and computation can also be attained.

As future scope, one can use a similar strategy that offers better flexibility. In this work parking price, time and location are taken as the crucial factors to determine an appropriate parking spot. Similarly, the score of reputation for both the parking owners and the drivers can be considered based on their evaluation to determine their access to the parking services. In these cases, establishing privacy preservation will be tasking job for updates, matching and calculation. Future work can also include fog node considerations in order to further decrease the cost of communication and computation. Moreover, privacy and reliability of the fog nodes should be also be taken into account while integrating with blockchain.

\section{References}

[1] Al-Turjman, F., \& Malekloo, A. (2019). Smart parking in IoT-enabled cities: A survey. Sustainable Cities and Society, 49, 101608.

[2] Aydin, I., Karakose, M., \& Karakose, E. (2017, April). A navigation and reservation based smart parking platform using genetic optimization for smart cities. In 2017 th International Istanbul Smart Grid and Cities Congress and Fair (ICSG) (pp. 120-124). IEEE.

[3] Ni, J., Zhang, K., Yu, Y., Lin, X., \& Shen, X. (2018). Privacy-preserving smart parking navigation supporting efficient driving guidance retrieval. IEEE Transactions on Vehicular Technology, 67(7), 6504-6517.

[4] Sadhukhan, P. (2017, September). An IoT-based E-parking system for smart cities. In 2017 International Conference on Advances in Computing, Communications and Informatics (ICACCI) (pp. 1062-1066). IEEE.

[5] Bock, F., Di Martino, S., \& Origlia, A. (2019). Smart parking: Using a crowd of taxis to sense on-street parking space availability. IEEE Transactions on Intelligent Transportation Systems, 21(2), 496-508.

[6] Kamel, K., Smys, S., \& Bashar, A. (2020). Tenancy Status Identification of Parking Slots Using Mobile Net Binary Classifier. Journal of Artificial Intelligence, 2(03), 146154. 
[7] Kayal, P., \& Perros, H. (2017, March). A comparison of IoT application layer protocols through a smart parking implementation. In 2017 20th Conference on Innovations in Clouds, Internet and Networks (ICIN) (pp. 331-336). IEEE.

[8] Ahmed, S., Rahman, M. S., \& Rahaman, M. S. (2019, March). A blockchain-based architecture for integrated smart parking systems. In 2019 IEEE International Conference on Pervasive Computing and Communications Workshops (PerCom Workshops) (pp. 177-182). IEEE.

[9] Barriga, J. J., Sulca, J., León, J. L., Ulloa, A., Portero, D., Andrade, R., \& Yoo, S. G. (2019). Smart parking: a literature review from the technological perspective. Applied Sciences, 9(21), 4569.

[10] Shakya, S., Pulchowk, L. N., \& Smys, S. (2020). Anomalies Detection in Fog Computing Architectures Using Deep Learning. Journal: Journal of Trends in Computer Science and Smart Technology March, 2020(1), 46-55.

[11] Abdulkader, O., Bamhdi, A. M., Thayananthan, V., Jambi, K., \& Alrasheedi, M. (2018, February). A novel and secure smart parking management system (SPMS) based on integration of WSN, RFID, and IoT. In 2018 15th Learning and Technology Conference (L\&T) (pp. 102-106). IEEE.

[12] Melnyk, P., Djahel, S., \& Nait-Abdesselam, F. (2019, October). Towards a Smart Parking Management System for Smart Cities. In 2019 IEEE International Smart Cities Conference (ISC2) (pp. 542-546). IEEE.

[13] Hilmani, A., Maizate, A., \& Hassouni, L. (2018). Designing and managing a smart parking system using wireless sensor networks. Journal of Sensor and Actuator Networks, 7(2), 24.

[14] Sungheetha, A., \& Sharma, R. (2020). Cost Effective Energy-Saving System in Parking Spots. Journal of Electronics, 2(01), 18-29. 